\title{
New excited states in the halo nucleus ${ }^{6} \mathrm{He}$
}

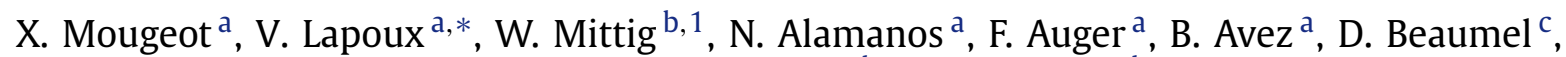

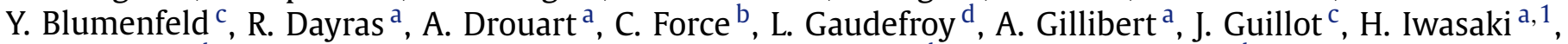 \\ T. Al Kalanee ${ }^{\mathrm{b}}$, N. Keeley ${ }^{\mathrm{e}}$, L. Nalpas ${ }^{\mathrm{a}}$, E.C. Pollacco ${ }^{\mathrm{a}}$, T. Roger ${ }^{\mathrm{b}}$, P. Roussel-Chomaz ${ }^{\mathrm{b}}$, D. Suzuki ${ }^{\mathrm{c}}$, \\ K.W. Kemper ${ }^{\text {f }}$, T.J. Mertzimekis ${ }^{\text {g, }}{ }^{,}$, A. Pakou ${ }^{g}$, K. Rusek $^{\mathrm{e}}$, J.-A. Scarpaci ${ }^{\mathrm{c}}$, C. Simenel ${ }^{\mathrm{a}}$, I. Strojek ${ }^{\mathrm{e}}$, \\ R. Wolski ${ }^{\text {h,i }}$
}

\footnotetext{
a CEA, Centre de Saclay, IRFU, Service de Physique Nucléaire, F-91191 Gif-sur-Yvette, France

b GANIL, Bld. Henri Becquerel, BP 5027, F-14021 Caen Cedex, France

c Institut de Physique Nucléaire, CNRS-IN2P3, F-91406 Orsay, France

d CEA, DAM, DIF, F-91297 Arpajon, France

e Department of Nuclear Reactions, National Centre for Nuclear Research, PL-00681, Warsaw, Poland

f Department of Physics, Florida State University, Tallahassee, FL 32306-4350, USA

${ }^{g}$ Department of Physics and HINP, University of Ioannina, GR 45110 Ioannina, Greece

h Flerov Laboratory of Nuclear Reactions, JINR, Dubna, RU-141980, Moscow region, Russia

i The Henryk Niewodniczanski Institute of Nuclear Physics, PL-31342, Kraków, Poland
}

\section{A R T I C L E I N F O}

\section{Article history:}

Received 7 March 2012

Received in revised form 21 September 2012

Accepted 7 October 2012

Available online 24 October 2012

Editor: V. Metag

\section{Keywords:}

${ }^{8} \mathrm{He}(\mathrm{p}, \mathrm{t})$

Transfer reaction

Borromean nucleus

Resonant states

\begin{abstract}
A B S T R A C T
The low-lying spectroscopy of ${ }^{6} \mathrm{He}$ was investigated via the 2-neutron transfer reaction $\mathrm{p}\left({ }^{8} \mathrm{He}, \mathrm{t}\right)$ with the ${ }^{8} \mathrm{He}$ beam delivered by the SPIRAL facility at $15.4 \mathrm{~A} \mathrm{MeV}$. The light charged particles produced by the direct reactions were measured using the MUST2 Si-strip telescope array. Above the known $2^{+}$state, two new resonances were observed: at $E^{*}=2.6 \pm 0.3 \mathrm{MeV}$ (width $\Gamma=1.6 \pm 0.4 \mathrm{MeV}$ ) and at $5.3 \pm 0.3 \mathrm{MeV}$ with $\Gamma=2 \pm 1 \mathrm{MeV}$. Through the analysis of the angular distributions, they correspond to a $2^{+}$state and to an $L=1$ state, respectively. These new states, challenging the nuclear theories, could be used as benchmarks for checking the microscopic inputs of the newly improved structure models, and should trigger development of models including the treatments of both core excitation and continuum coupling effects.
\end{abstract}

(c) 2012 Elsevier B.V. All rights reserved.
These last 30 years, the use of radioactive beams to produce nuclei far from the valley of stability has led to the discovery of a large variety of new phenomena, which were not expected in the nuclear structure theories tested on the stable nuclei: alphacluster like, halo or neutron-skin structures, low-lying resonant states have been observed in the light nuclei close to the neutron drip-line $[1,2]$. The shell structure has also been found deeply modified from the picture established close to the $\beta$-stability line, with the apparition of new magic numbers [3]. These new features have been triggering intensive theoretical developments to explain the change in our usual nuclear structure conceptions. The recent highlights in our field have been the findings of the role

\footnotetext{
* Corresponding author.

E-mail address: valerie.lapoux@cea.fr (V. Lapoux).

1 Present address: NSCL, Michigan State University, USA.

2 Present address: University of Athens, Greece.
}

played by the three-nucleon interaction (TNI) in the nuclear spectra of the light nuclei [4] and in the drip-line location [6,5] as well as the influence of the spin-isospin tensor term in the shell level ordering of the states [3] and the effects of the continuumcoupling (CC) between bound, resonant and scattering states in the low-lying spectra of the weakly-bound nuclei [7]. To understand the weak binding features of the light nuclei close to the drip-line, these CC effects have been investigated within various frameworks, the Gamow Shell Model (GSM) [7-9], the Continuum Shell Model CSM [10] or the Complex Scaled Cluster Orbital Shell Model (COSM) [11]. However, if these nuclear models are successful in predicting the characteristics of the ground or first well-studied excited states of the p-shell exotic nuclei, they disagree for the predictions of the other low-lying excited states. The discrepancies between theories question the validity of the microscopic inputs used for the description of the nuclear interactions, the various techniques adopted for the treatment of the many-body correlations, and their interplay with the CC effects. 

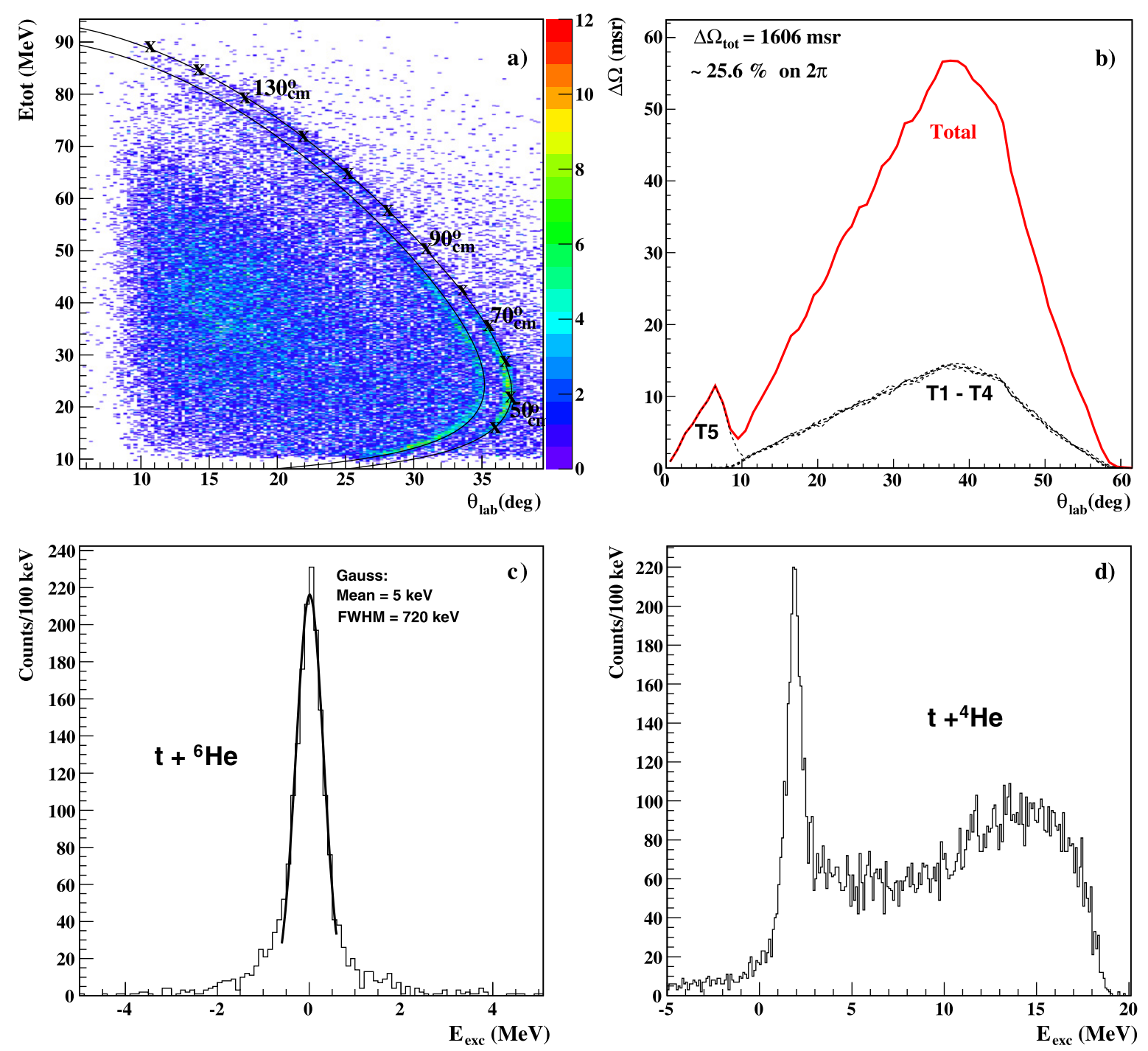

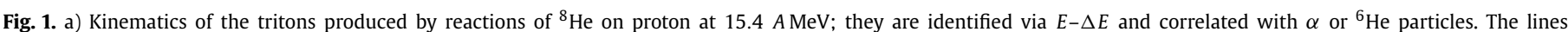

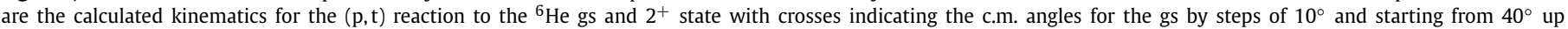

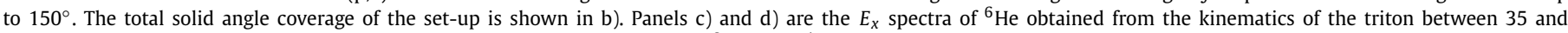

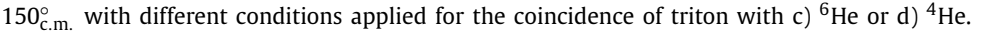

In this context, the low-lying spectroscopy of the light exotic nuclei represents a testing ground to constrain the models and to check their assumptions. It is the purpose of this Letter to present our experimental study of the low-lying positive parity excited states of the ${ }^{6} \mathrm{He}$ nucleus and to compare it to the predictions of the most recent nuclear theories. ${ }^{6} \mathrm{He}$ has neutron thresholds located at low energy $\left(S_{\mathrm{n}}=1.87\right.$ and $\left.S_{2 \mathrm{n}}=0.97 \mathrm{MeV}\right)$ and no bound excited state. The first excited state is a $2^{+}$at $1.8 \mathrm{MeV}$ $(\Gamma=113 \mathrm{keV})[12] .{ }^{6} \mathrm{He}$ is now well known as a halo nucleus [13, 2] and the 2n-halo structure was intensively investigated within few-body models [14,2], but the positions, spin and parities of the resonant states above $1.8 \mathrm{MeV}$ remained to be determined. On the theoretical side, various calculations $[14,11,4]$ or No-Core Shell Model (NCSM) with TNI [15], indicated that a series of $2_{2}^{+}, 1_{1}^{+}$, $0_{1}^{+}$states should exist above the $2_{1}^{+}$state and below the tritontriton threshold $S_{\mathrm{t}+\mathrm{t}}$ at $12.3 \mathrm{MeV}$, but they disagree on the energies of these states. Experimentally, the main results were obtained via transfer reactions, which indicated resonances below $S_{t+t}$ and broad resonances above. From the ${ }^{7} \mathrm{Li}\left({ }^{6} \mathrm{Li},{ }^{7} \mathrm{Be}\right){ }^{6} \mathrm{He}$ reaction [16], a $2^{+}$state was indicated at $5.6 \mathrm{MeV}$ with a $\Gamma=10.9 \mathrm{MeV}$ width and structures possibly $(1,2)^{-}$at $14.6(\Gamma=7.4) \mathrm{MeV}$, and at
$23.3(\Gamma=14.8) \mathrm{MeV}$; a broad one $(\Gamma=4)$ at $4 \mathrm{MeV}$ was reported in Ref. [17], and at $18 \mathrm{MeV}(\Gamma=7.7)$ in Ref. [18]. From the ${ }^{6} \mathrm{Li}\left(\mathrm{t},{ }^{3} \mathrm{He}\right){ }^{6} \mathrm{He}$ reaction resonance-like structures were seen at 7.7, 9.9 MeV and at $5(\Delta L=1)$ and $15 \mathrm{MeV}$ [19]. No resonance except the $2^{+}$was indicated from the ${ }^{6} \mathrm{He}\left(\mathrm{p}, \mathrm{p}^{\prime}\right)$ scattering at $40.9 \mathrm{~A} \mathrm{MeV} \mathrm{[13].} \mathrm{None} \mathrm{of} \mathrm{these} \mathrm{experiments} \mathrm{was} \mathrm{successful} \mathrm{to} \mathrm{de-}$ termine precisely the energy and width of the expected resonant states. In a recent experiment at the GANIL facility, the scattering and $1 \mathrm{n}$-transfer reaction of the ${ }^{8} \mathrm{He}$ SPIRAL beam on proton [20] were studied. The $(\mathrm{p}, \mathrm{t})$ to the ${ }^{6} \mathrm{He}$ ground state $(\mathrm{gs})$ and $2_{1}^{+}$excited state were also measured [21], with cross sections of the order of $1 \mathrm{mb} / \mathrm{sr}$. This $2 \mathrm{n}$ transfer appeared to be a good probe to populate the possible excited states of ${ }^{6} \mathrm{He}$ with sufficient yields, taking advantage of the low energy and high intensity of the ${ }^{8} \mathrm{He}$ SPIRAL beam.

We report here the results of the $(\mathrm{p}, \mathrm{t})$ experiment carried out with the ${ }^{8} \mathrm{He}$ beam accelerated at $15.4 \mathrm{AMeV}$, and using an improved set-up to measure at forward angles the triton-particle correlations in the exit channel: triton with either ${ }^{6} \mathrm{He}$ in case of the $(\mathrm{p}, \mathrm{t})^{6} \mathrm{He}_{g s}$, or with the ${ }^{4} \mathrm{He}$ particle produced in the decay of the excited states of the ${ }^{6} \mathrm{He}$ nucleus into ${ }^{4} \mathrm{He}+2 \mathrm{n}$. The 

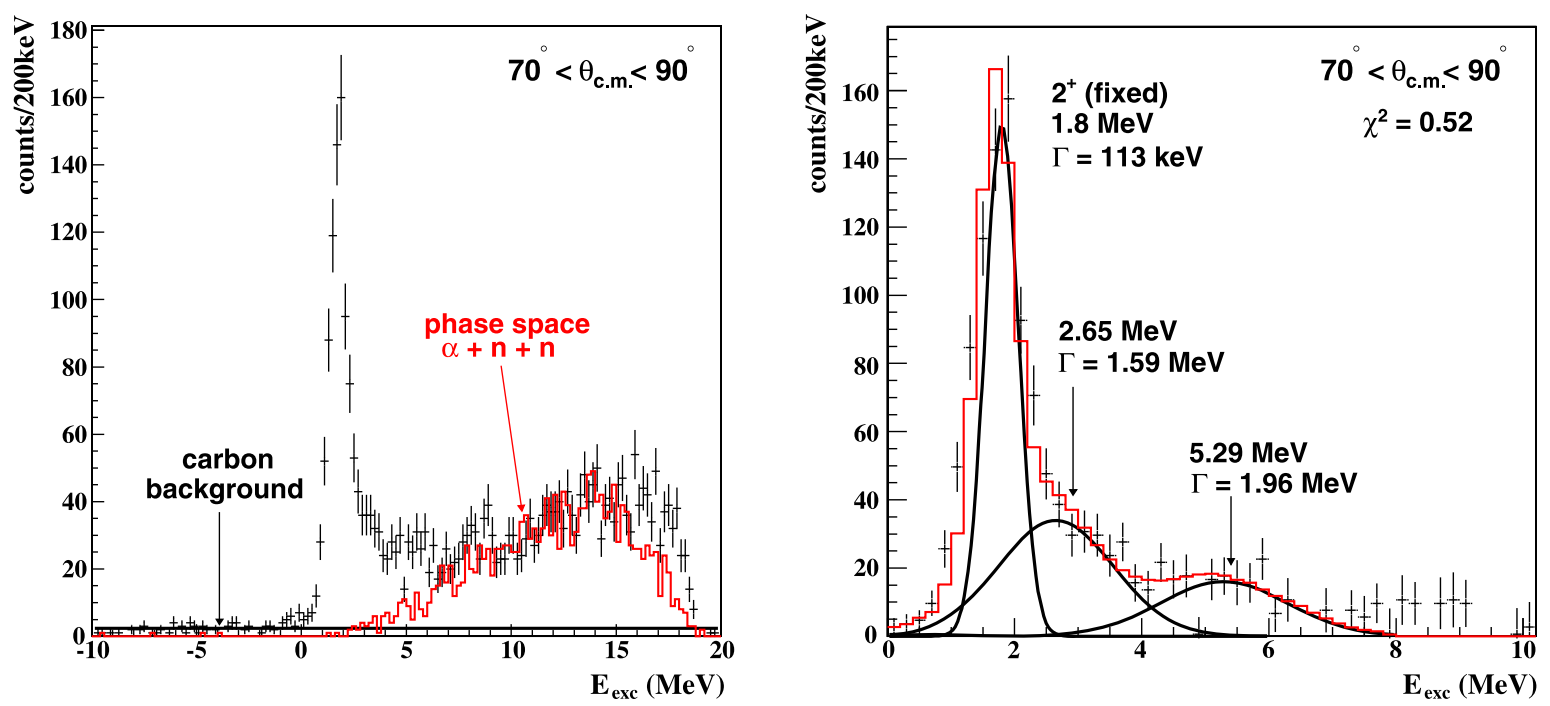

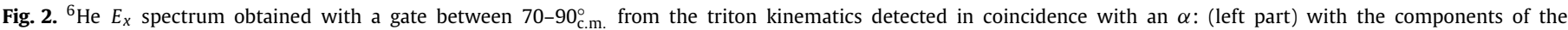

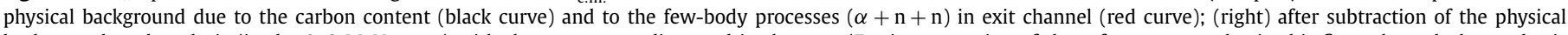

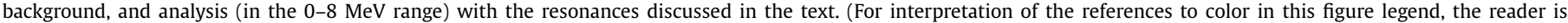
referred to the web version of this Letter.)

beam had no contaminant, and a mean intensity of $1.8 \times 10^{4} / \mathrm{s}$. The target was a $50 \mu \mathrm{m}$-thick $\left(4.48 \mathrm{mg} / \mathrm{cm}^{2}\right)$ foil of polypropylene $\left(\mathrm{CH}_{2}\right)_{n}$. Two beam tracking detectors CATS [22] were mounted upstream of the target to reconstruct the incident trajectories on the target. The measured beam spot sizes were 4 and $5 \mathrm{~mm}$ (FWHM) in horizontal and vertical directions, respectively, and of the order of $0.5^{\circ}$ (FWHM) for the angle distributions. The identification of the particles, reconstruction of the trajectory and measurement of the energies were done by combining five telescopes of the new MUST2 Si-strip array [23] at forward angles: four modules were arranged in a square wall located $15 \mathrm{~cm}$ downstream of the target, and the fifth one was at $30 \mathrm{~cm}$. The light charged particles protons $(p)$, deutons $(d)$ and tritons $(t)$ produced by the elastic, $\left(p, p^{\prime}\right)$ and $1 \mathrm{n}$ and $2 \mathrm{n}$ transfer reactions were measured in the wall, in coincidence with the ejectiles focused at forward angles in the 5th telescope placed behind a plastic scintillator. The first stage of each telescope was a $300 \mu \mathrm{m}$ thick Si-strip detector with an active area of $10 \times 10 \mathrm{~cm}^{2}$ and $128(\mathrm{X}, \mathrm{Y})$ strips, measuring the energy loss $\Delta E$, the time of flight (ToF, with reference to the second CATS) and the position. The angular resolution at $15 \mathrm{~cm}$ from the target is $0.3^{\circ}$; the energy resolution of the Si-strips is $40 \mathrm{keV}$ at $5.5 \mathrm{MeV}$. The residual energy $E$ was measured by the second stage, a $4 \mathrm{~cm}$-thick CsI-crystal detector. Depending on their energies, the particles are identified using the standard correlation techniques between $\Delta E$ and ToF, and $\Delta E$ versus $E[23]$.

The kinematical characteristics of the triton, total kinetic energy and scattering angle in laboratory (lab.) frame, were obtained event by event. By considering the events with $\mathrm{t}+{ }^{6} \mathrm{He}$ or $\mathrm{t}+\alpha$ coincidences, the ${ }^{8} \mathrm{He}(\mathrm{p}, \mathrm{t})$ reactions were fully reconstructed, and the excitation energy $\left(E_{x}\right)$ spectra of ${ }^{6} \mathrm{He}$ were deduced via missing mass method from the relativistic kinematics of the triton. We consider here the tritons identified in MUST2 via $E-\Delta E$. Their energies are above $11 \mathrm{MeV}$, with $(\mathrm{p}, \mathrm{t})$ kinematics corresponding to angles in the center of mass (c.m.) frame from $35^{\circ}$ up to $150^{\circ}$. The kinematics and the deduced $E_{x}$ spectra of ${ }^{6} \mathrm{He}$ are presented in Fig. 1. These events were collected with a total number of $1.52 \times 10^{9}{ }^{8} \mathrm{He}$. The contributions of possible $\alpha$-t coincidences due to reactions on the carbon of the $\left(\mathrm{CH}_{2}\right)_{n}$ target were measured with a pure carbon target. The analysis of the $E_{x}$ spectra obtained for different angular ranges showed that the physical background due to the reactions on carbon is constant in our domain of excitation energies. It is modelled by the thick line shown in the spectra in Figs. 2-3. The physical background was also estimated. It is due to many-body kinematical effects of the processes producing the same particles as the $(\mathrm{p}, \mathrm{t})$ reaction, $\alpha$ and $\mathrm{t}$ in the final state, but without involving an excited state of ${ }^{6} \mathrm{He}$. Only 3 processes may contribute, corresponding to the detection of $t+\alpha$ in the final state, either with a 3-body kinematics: (i) $\mathrm{t}+{ }^{5} \mathrm{He}+\mathrm{n}$ with the decay ${ }^{5} \mathrm{He} \rightarrow \alpha+\mathrm{n}$, (ii) $\mathrm{t}+\alpha+\mathrm{n}^{2}$ with 2 correlated neutrons, or (iii) a 4-body: $\mathrm{t}+\alpha+\mathrm{n}+\mathrm{n}$ with 2 uncorrelated neutrons. The main source is the contribution of the ${ }^{6} \mathrm{He}^{*}$ decay into $\alpha+\mathrm{n}+\mathrm{n}$.

We adopted the Breit-Wigner parametrization to define the distribution of a resonance with the energy $E_{R}$ and intrinsic width $\Gamma_{R}$ :

$f(E)=\frac{1}{\pi} \frac{\Gamma_{R} / 2}{\left(\mathrm{E}-\mathrm{E}_{R}\right)^{2}+\left(\Gamma_{R} / 2\right)^{2}}$.

The possible resonances were modelled as Breit-Wigner shapes of width $\Gamma$ folded with a Gaussian function, to take into account the spreading due to the experimental resolution, estimated of the order of 610 up to $720 \mathrm{keV}$ (FWHM). The known resonance $2_{1}^{+}$was obtained at 1.8 (2) $\mathrm{MeV}, \Gamma=0.1 \pm 0.2 \mathrm{MeV}$ consistent with the literature, and then fixed in the search at $1.8 \mathrm{MeV}$ with $\Gamma=113 \mathrm{keV}$. The parameters of the other resonances were fitted, and the normalization of all the peaks and resonances were free to vary.

A structure can be considered as a resonant nuclear state if its parameters (position and width) are conserved within error bars, whatever the angular slice examined. To exhibit the fit of the resonances, we first show in Fig. 2 an example of the $E_{x}$ spectrum obtained for the angular range $70-90_{\mathrm{c} . \mathrm{m}}^{\circ}$, with full statistics (left part) and analyzed after background subtraction. This operation was only the preliminary step to locate more easily the resonances, in the search range between 0 and $8 \mathrm{MeV}$. Two new possible states are indicated between 1.8 and $8 \mathrm{MeV}$. Above $8 \mathrm{MeV}$ the structures are embedded in the physical background and no resonance parameter could be extracted consistently. The complete analysis, to confirm the existence of the resonances, was done by fitting the $E_{x}$ spectra with the full data set (no background subtraction), for various angular slices and in the $0-20 \mathrm{MeV}$ range, so as to take 

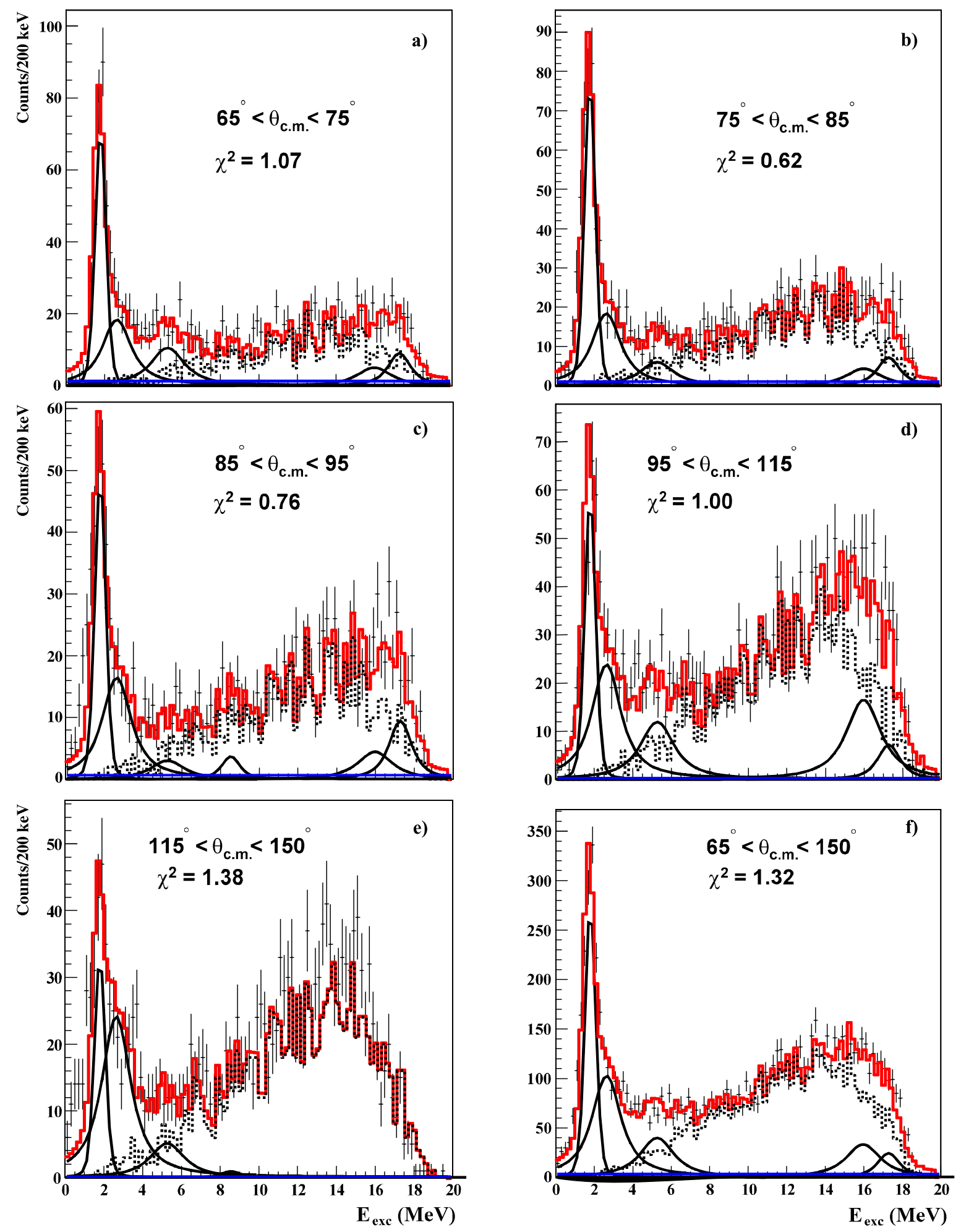

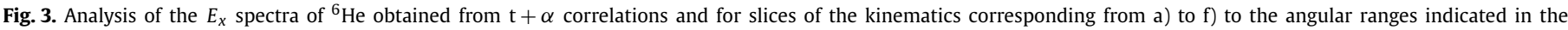

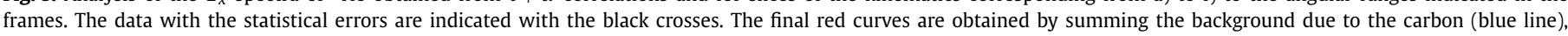

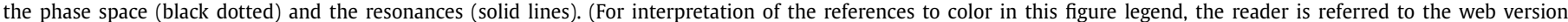
of this Letter.)

into account the effect of the physical background at high energies. The resonance parameters were extracted in a kinematical domain for which the best excitation energy resolutions are obtained (less than $720 \mathrm{keV}$ FWHM) with significant statistics: between 65 and $150_{\mathrm{c} . \mathrm{m} \text {. }}^{\circ}$ the $E_{X}$ spectra obtained for various small c.m. gates (range of $10_{\mathrm{c} . \mathrm{m} .}^{\circ}$ ) were analyzed to check the consistency of the extracted parameters of the possible resonances, and the number of these states was also varied and checked. These $E_{x}$ spectra obtained by applying the c.m. gates: $[65 ; 75]^{\circ},[75 ; 85]^{\circ},[85 ; 95]^{\circ}$, $[95 ; 115]^{\circ}$ and $[115 ; 150]^{\circ}$ are shown in Fig. 3 with the total slice 

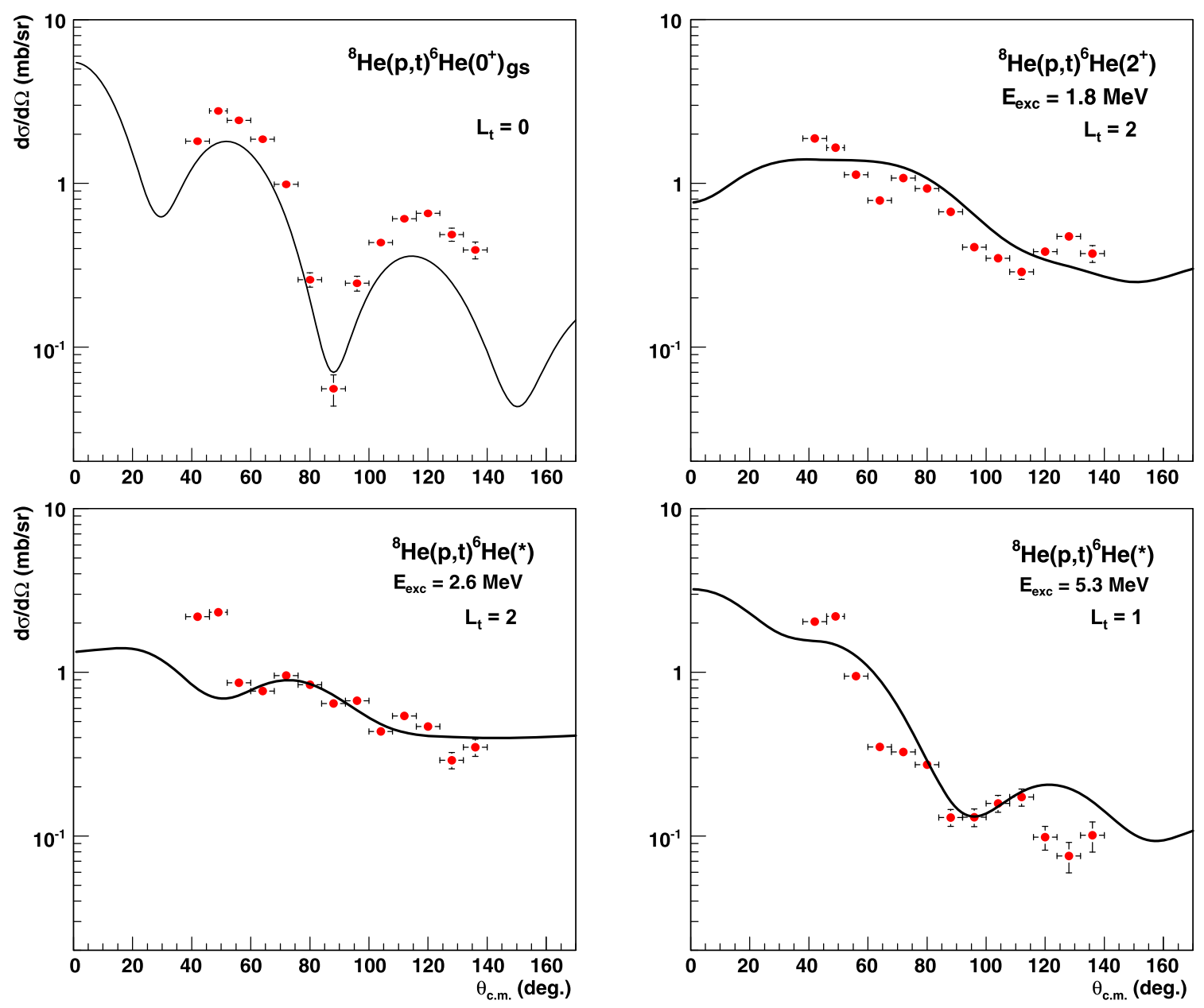

Fig. 4. Experimental cross sections for ${ }^{8} \mathrm{He}(\mathrm{p}, \mathrm{t})$ at $15.4 \mathrm{AMeV}$. The calculated curves (top panels: 2-step CRC, bottom: 1 -step CRC) are described in the text.

for $[65 ; 150]^{\circ}$. The background effects (black dotted curve in Fig. 3) are modelled with a Monte Carlo simulation including the phase space calculations and the experimental response of the set-up. At excitation energies above $S_{t+t}$, the only way to reproduce the shape of the data is to introduce two broad structures located at 16 (width $2 \mathrm{MeV}$ ) and $17.3 \mathrm{MeV}$ (width $1.1 \mathrm{MeV}$ ) in addition to the phase space contributions. Being at the limit of the kinematical efficiency, their positions cannot be determined precisely. However they are consistent with the broad structures reported in previous experiments $[18,19]$.

The resonances in search and the total physical background contributions were included in the calculated red curve in Fig. 3. The $\chi^{2}$ minimization between the data and the calculated curve was considered for the energy range between 0 and $20 \mathrm{MeV}$. A structure observed around $8 \mathrm{MeV}$ was not considered as a state since it was observed for only one slice. New resonances were found: at $2.6 \pm 0.3 \mathrm{MeV}$ with an intrinsic width $\Gamma=1.6 \pm 0.4 \mathrm{MeV}$ and at $5.3 \pm 0.3 \mathrm{MeV}$ with $\Gamma=2 \pm 1 \mathrm{MeV}$.

Now, considering all the events with a triton detected in MUST2 and no forward coincidence, the angular distributions were deduced for the $(\mathrm{p}, \mathrm{t})$ to the gs, the known $2_{1}^{+}$and the new excited states of ${ }^{6} \mathrm{He}$. The statistical error is included within the points of Fig. 4. The systematical errors due to the target thickness, the normalization, the efficiency and the subtraction of the background were estimated to the level of $11 \%$ in the case of the resonant states, and $9 \%$ for the bound state in exit channel (total error bar). We have obtained distributions to the new states, and the extension of the $(\mathrm{p}, \mathrm{t}) 0^{+}, 2_{1}^{+}$up to $140_{\mathrm{c} . \mathrm{m} \text {. }}^{\circ}$. First, we compare the data for the $(\mathrm{p}, \mathrm{t})$ distributions to the gs and to the $2_{1}^{+}$state at $15.4 \mathrm{AMeV}$ with the analysis done in the coupled-reactionchannel (CRC) framework. The previous data set at $15.6 \mathrm{~A} \mathrm{MeV}$ was obtained only for the gs and $2_{1}^{+}$states, below $80_{\mathrm{c} . \mathrm{m} .}^{\circ}[20,21]$. It was described consistently within CRC calculations, the spectroscopic factors (SF) of the ${ }^{8} \mathrm{He}_{g s}$ with respect to ${ }^{7} \mathrm{He}_{3 / 2^{-}}$gs and ${ }^{6} \mathrm{He}\left(0^{+}\right)$and ${ }^{6} \mathrm{He}\left(2^{+}\right)$were extracted, the $\mathrm{C}^{2} \mathrm{~S}$ values were 2.9 , 1.0 and 0.014 , respectively, with error bars of the order of $30 \%$. The agreement between the present data set and the previous CRC curves confirms the extracted SFs, and the gs structure of ${ }^{8} \mathrm{He}$ interpreted as a mixing between the $\left(1 \mathrm{p}_{3 / 2}\right)^{4}$ and $\left(1 \mathrm{p}_{3 / 2}\right)^{2}\left(1 \mathrm{p}_{1 / 2}\right)^{2}$ configurations. This corroborates the $\alpha+4 \mathrm{n}$ calculations [25] and the recent dineutron cluster structure discussed by the AMD theory [26].

Two-step CRC calculations including the new states of ${ }^{6} \mathrm{He}$ are needed to fully interpret the new data. This would require to complete the previous CRC framework discussed in Ref. [21] and to include consistently the two new states as shown in Fig. 5. By comparison between the data and the calculations, the SF of the new states could be inferred. However, these calculations remain complicated due to the interplay of the new channels. The development of this framework and the extraction of the SF for the new states is in progress and will be presented in a forthcoming article [24]. Here we can simplify the analysis to deduce the transferred angular momentum $L_{t}$ of the new states, by comparing the shape of the curves for various $L_{t}$ values to the data. The 2 curves presented in Fig. 4 (top panels) are examples of $L_{t}=0,2$ within 2-step calculations. $L_{t}=0$ can be excluded for the new 


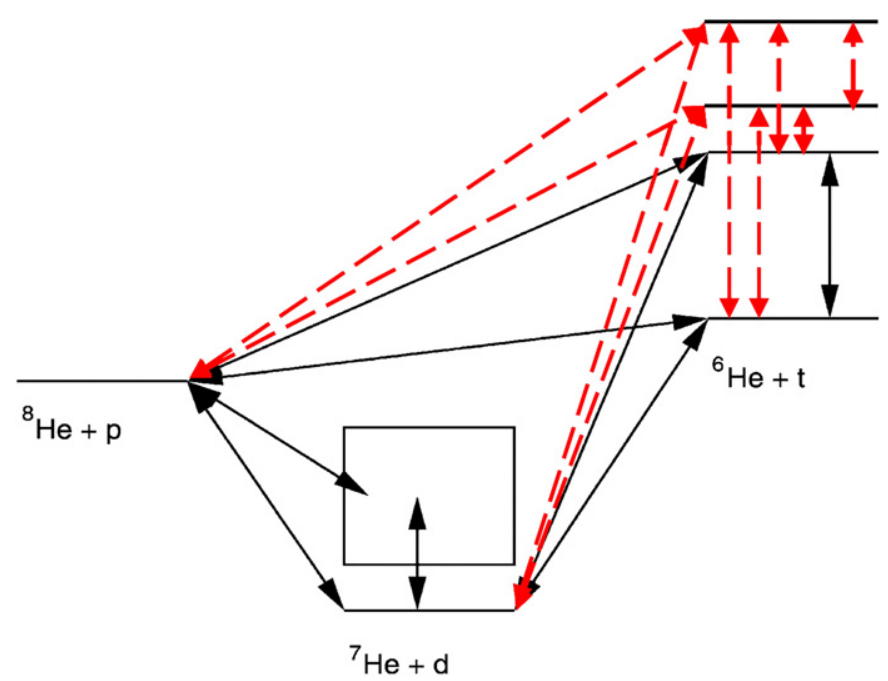

Fig. 5. Schematic of the coupling scheme needed for the 2-step CRC calculations: the black lines show the channels included for the calculations of $(p, t)$ to the gs and $2_{1}^{+}$state of ${ }^{6} \mathrm{He}$; the red ones are the channels required for the new states. (For interpretation of the references to color in this figure legend, the reader is referred to the web version of this Letter.)

states, $L_{t}=2$ shape is consistent only for the $2.6 \mathrm{MeV}$ state. We have also considered various $L_{t}$ values for the one-step $0^{+} 2 \mathrm{n}$-pair transfer between the $0^{+}$gs of ${ }^{8} \mathrm{He}$ and the new excited states. The curves in agreement with the shape of the cross sections are given in Fig. 4: the $(\mathrm{p}, \mathrm{t})$ distribution to the $2.6 \mathrm{MeV}$ state is reproduced with an $L_{t}=2$; this state is a $2^{+}$. The state at $5.3 \mathrm{MeV}$ corresponds to an $L_{t}=1$. Of course, such limited 1-step transfer calculation can produce only the natural parity $1^{-}$corresponding to $L_{t}=1$. However within 2 -step processes for the $(\mathrm{p}, \mathrm{t})$, the neutrons can be transferred sequentially, and, in this future complete framework, this $L_{t}=1$ state may correspond to a $1^{+}$.

These resonances are compared to the calculations from various theoretical frameworks: the few-body model [14], the QMC using UIX and TNI (IL2 force) [4], NCSM with TNI (TM99) [15], the models treating explicitly the CC of bound and scattering states like CSM [10] and GSM [8,9], and the Complex Scaled COSM [11]. The inclusion of TNI force which is crucial to have the correct features for the binding energy and the $2_{1}^{+}$state is not sufficient to predict correctly the other states, strongly affected by the CC. As can be seen in Fig. 6 , the models including the $\mathrm{CC}[8,11]$ provide a better description of the $2^{+}$states, found closer to the gs than in the other models. The important feature of these models is their realistic treatment of the coupling to the continuum: the two neutrons of the halo can interact with each other and be excited to the continuum states. However, a complete understanding of both the position and width of the new states is not reached with the present version of the modern theories. Several effects may be needed to improve the description of ${ }^{6} \mathrm{He}$, such as the excitations of the ${ }^{4} \mathrm{He}$ core, considered as inert in most calculations. A promising approach was developed recently: the role of the particle-hole excitations of ${ }^{4} \mathrm{He}$ is now taken into account in the coupled-cluster calculations of ${ }^{6} \mathrm{He}$ states [27]. These effects may provide new interesting findings for the properties of the states located above the $S_{\mathrm{n}}$ threshold.

In conclusion, the results obtained via the ${ }^{8} \mathrm{He}(\mathrm{p}, \mathrm{t})$ reactions are the most complete ones collected for the low-lying spectroscopy of ${ }^{6} \mathrm{He}$, with the evidence of two new resonances and the measurement of a whole set of angular distributions. The new states at 2.6 (3) and at 5.3 (3) $\mathrm{MeV}$ were found consistent with a $2^{+}$ and an $L_{t}=1$ state, respectively. They provide new constraints for

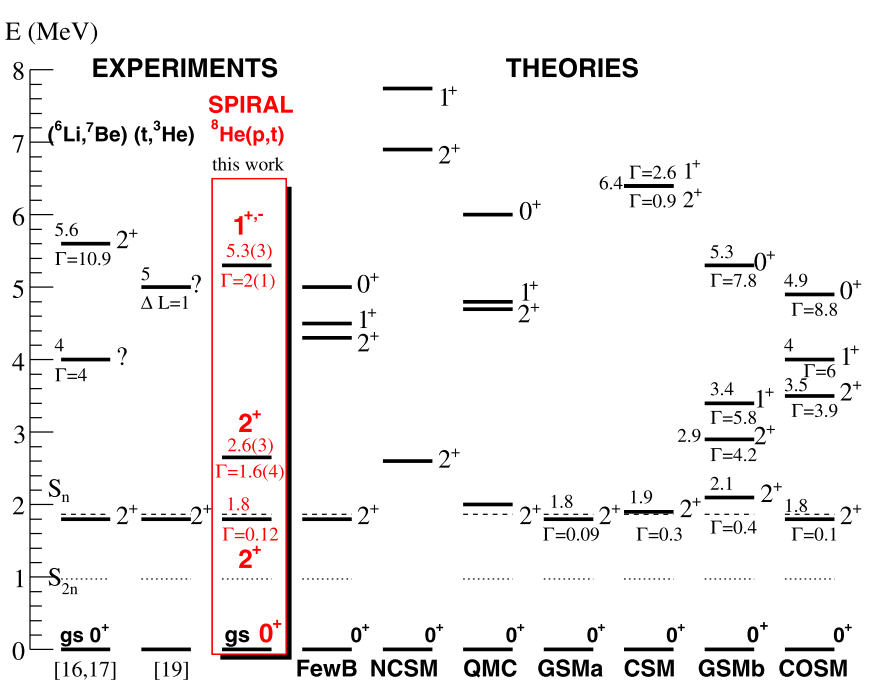

Fig. 6. Spectroscopy of ${ }^{6} \mathrm{He}$ : comparison between our new results with the previous experiments and with several theories, few-body model (FewB) [14], QMC [4], NCSM [15], CSM [10], GSMa [9], GSMb [8], and the COSM [11].

the nuclear models. More generally, resonant states of the light exotic nuclei could represent benchmark data sets for the future approaches, expected to combine the treatment of the realistic interaction developed on the 3-body forces with the many-body correlations between valence nucleons and continuum effects [9].

\section{Acknowledgements}

We wish to thank the SPEG staff, P. Gangnant and J.-F. Libin, and the GIP team at GANIL, for their help in the preparation of the experiment.

\section{References}

[1] I. Tanihata, et al., Prog. Part. Nucl. Phys. 35 (1995) 505.

[2] B. Jonson, Phys. Rep. 389 (2004) 1.

[3] T. Otsuka, et al., Phys. Rev. Lett. 87 (2001) 082502; T. Otsuka, et al., Phys. Rev. Lett. 95 (2005) 232502.

[4] S.C. Pieper, R.B. Wiringa, J. Carlson, Phys. Rev. C 70 (2004) 054325.

[5] T. Otsuka, et al., Phys. Rev. Lett. 105 (2010) 032501.

[6] G. Hagen, et al., Phys. Rev. C 80 (2009) 021306.

[7] N. Michel, W. Nazarewicz, M. Ploszajczak, K. Bennaceur, Phys. Rev. Lett. 89 (2002) 042502;

N. Michel, W. Nazarewicz, M. Ploszajczak, K. Bennaceur, Phys. Rev. C 67 (2003) 054311.

[8] G. Hagen, M. Hjorth-Jensen, J.S. Vaagen, Phys. Rev. C 71 (2005) 044314.

[9] N. Michel, W. Nazarewicz, M. Ploszajczak, Phys. Rev. C 82 (2010) 044315.

[10] A. Volya, V. Zelevinsky, Phys. Rev. Lett. 94 (2005) 052501.

[11] T. Myo, K. Kato, K. Ikeda, Phys. Rev. C 76 (2007) 054309.

[12] D.R. Tilley, et al., Nucl. Phys. A 708 (2002) 3.

[13] A. Lagoyannis, et al., Phys. Lett. B 518 (2001) 27.

[14] B.V. Danilin, et al., Phys. Rev. C 55 (1997) 577.

[15] P. Navrátil, W.E. Ormand, Phys. Rev. C 68 (2003) 034305.

[16] J. Jänecke, et al., Phys. Rev. C 54 (1996) 1070.

[17] S. Nakayama, et al., Phys. Rev. Lett. 85 (2000) 262

[18] H. Akimune, et al., Phys. Rev. C 67 (2003) 051302.

[19] T. Nakamura, et al., Phys. Lett. B 493 (2000) 209; Eur. Phys. J. A 13 (2002) 33.

[20] F. Skaza, et al., Phys. Lett. B 619 (2005) 82; F. Skaza, et al., Phys. Rev. C 73 (2006) 044301

[21] N. Keeley, et al., Phys. Lett. B 646 (2007) 222.

[22] S. Ottini, et al., Nucl. Instrum. Methods Phys. Res. A 431 (1999) 476.

[23] E. Pollacco, et al., Eur. Phys. J. A 25 (2005) 287.

[24] X. Mougeot, V. Lapoux, et al., Phys. Rev. C, submitted for publication.

[25] K. Hagino, N. Takahashi, H. Sagawa, Phys. Rev. C 77 (2008) 054317.

[26] Y. Kanada-En'yo, Phys. Rev. C 76 (2007) 044323.

[27] G.R. Jansen, M. Hjorth-Jensen, G. Hagen, T. Papenbrock, Phys. Rev. C 83 (2011) 054306. 